\title{
Diet and Lifestyle as Risk Factors for Carotid Artery Disease: A Prospective Cohort Study
}

\author{
Anna Johansson ${ }^{a, b}$ Stefan Acosta ${ }^{a, c}$

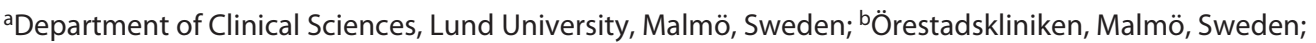 \\ 'Vascular Center, Department of Cardiothoracic and Vascular Surgery, Skåne University Hospital, Skåne, Sweden
}

\section{Keywords}

Stroke $\cdot$ Carotid artery disease $\cdot$ Atherosclerosis .

Epidemiology $\cdot$ Diet

\begin{abstract}
Background: Stroke is one of the leading causes of death, and out of all stroke cases, $10-15 \%$ originate from a previously asymptomatic stenosis in the internal carotid artery. Aims: The aim of the study was to investigate whether dietary and lifestyle habits were associated with future risk of incident carotid artery disease (CAD). Methods: Baseline examinations on middle-aged individuals $(n=30,447)$ in the Malmö Diet and Cancer study (MDCS), a prospective cohort study, took place between 1991 and 1996 in Malmö, Sweden. Individuals with cardiovascular disease and diabetes mellitus were excluded at baseline, resulting in a total study population of 25,952 patients. Information on dietary intake was gathered through a 7-day food diary, a detailed questionnaire, and a 1-h interview. A diet quality index was calculated from adherence to recommended intake of 6 dietary components (saturated fat, polyunsaturated fat, fish and shellfish, fiber, vegetables and fruit, and sucrose). Individuals with a first registered diagnosis of CAD were identified from
\end{abstract}

karger@karger.com www.karger.com/ced

Karger $\stackrel{\text { ' }}{5}$

GOPEN ACCESS
(C) 2020 The Author(s)

Published by S. Karger AG, Basel

This is an Open Access article licensed under the Creative Commons Attribution-NonCommercial-4.0 International License (CC BY-NC) (http://www.karger.com/Services/OpenAccessLicense), applicable to the online version of the article only. Usage and distribution for commercial purposes requires written permission. the Swedish National Patient register. Results: During a median follow-up of 21.8 years, 469 participants (1.8\%) developed CAD. The diagnosis of incident CAD was validated and confirmed in $99 \%$ of a random sample of 100 individuals. Higher intake of vegetables and fruit was associated with a trend of decreased risk of CAD in a Cox regression analysis (hazard ratio of $0.76,95 \%$ confidence interval $0.56-1.03 ; p=$ 0.080 ). Conclusions: In conclusion, the present study found a trend toward a protective effect of higher intake of vegetables and fruit against incident CAD. More prospective studies investigating the association between diet and CAD and stroke are needed in order to give firm recommendations.

(c) 2020 The Author(s)

Published by S. Karger AG, Basel

\section{Introduction}

In Europe with its population of approximately 740 million inhabitants [1], around 1.6 million stroke cases occur yearly. When it comes to mortality, stroke is the second most common cause of death in Europe, accounting for 1 million deaths each year [2]. More than half of stroke survivors are dependent on others to be able to 
Fig. 1. Descriptive flow diagram of study participants, dietary data, and exclusions. Some individuals had multiple exclusion criteria. CAD, carotid artery disease; $\mathrm{CoAD}$, coronary artery disease; PAD, peripheral artery disease.

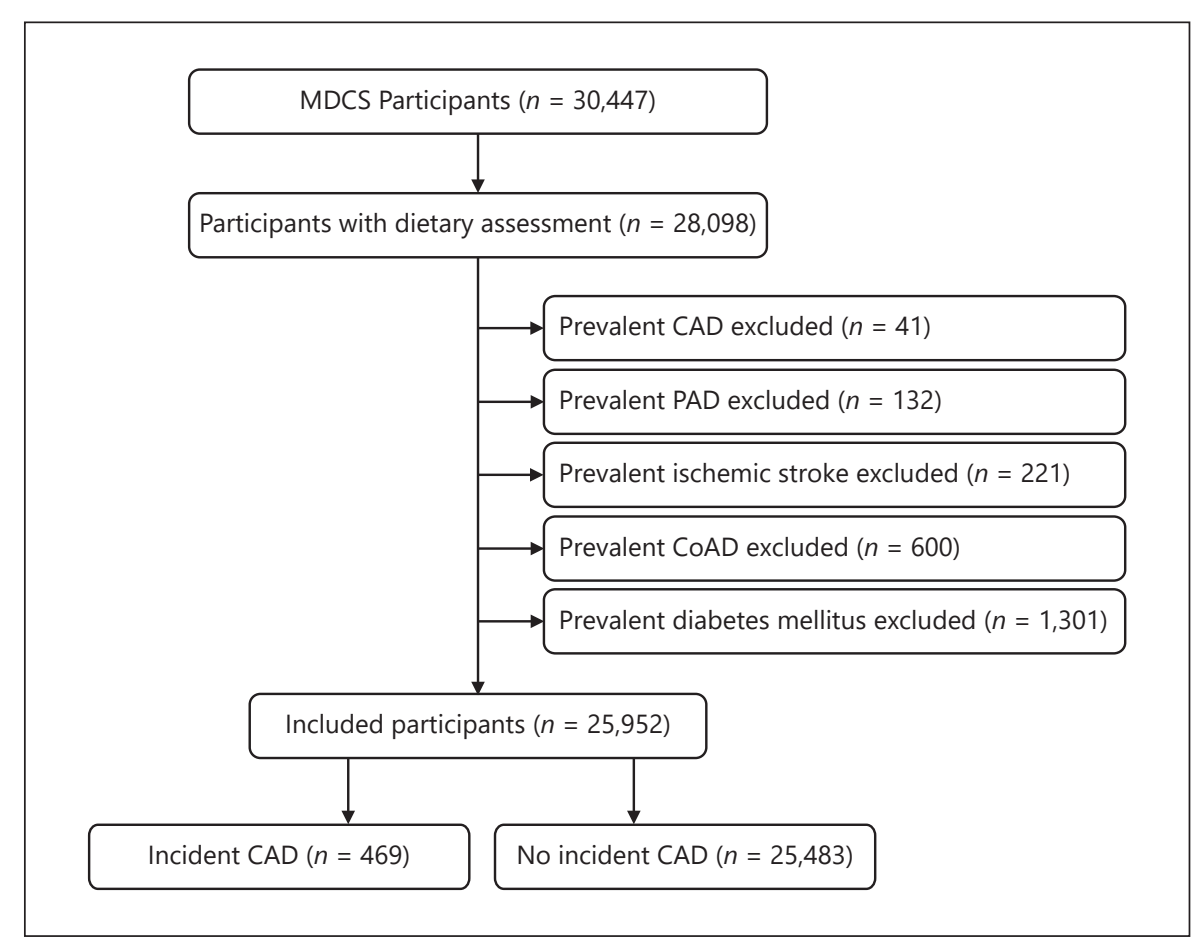

perform their activities of daily living [3]. Stroke generates enormous costs for health care systems in the European Union, in total around 45 billion Euros every year [2].

Overall, $10-15 \%$ of all stroke cases originate from a previously asymptomatic stenosis $(>50 \%)$ in the internal carotid artery [4]. In addition, presence of atherosclerosis in the internal carotid artery has been shown to be an independent risk factor for cardiovascular (CV) events [5].

Guidelines for managing CV diseases [6] often highlight the importance of healthy dietary habits and other lifestyle factors, being modifiable risk factors for CV disease. Lack of a high intake of vegetables and fruit is a contributing risk factor to the development of hypertension and its complications [7]. However, the association between nutrients and lifestyle factors and development of carotid artery disease (CAD) has been scarcely studied.

The purpose of this population-based prospective longitudinal study was to evaluate whether dietary habits and lifestyle are associated with future risk of incident CAD. The results can potentially improve our understanding of the role of diet in CAD and ischemic stroke. The study hypothesis was that there would be an inverse association between healthy diet and incident CAD.

\section{Methods}

Study Population and Data Collection

Men born 1923-1945 and women born 1923-1950 living in Malmö were eligible to enter the Malmö Diet and Cancer study (MDCS) and baseline examinations were carried out between 1991 and 1996 [8]. Individuals who underwent dietary assessment were included, whereas individuals with prevalent CV disease were excluded. The cohort was designed to study dietary habits, and individuals who more likely might have changed the dietary habits during follow-up, such as patients with known diabetes mellitus and CV disease, were excluded at baseline (Fig. 1).

Diet Assessment Method and Diet Quality Index

Information on dietary habits was collected through a 7-day food diary, where the participants recorded their food intake at lunch and dinner meals and intake of cold beverages, together with a 168-item food frequency questionnaire, where frequency and portion size of foods regularly consumed during the past year were documented [9]. The participants underwent a 1-h interview, where further details were collected. A diet quality index has previously been developed and validated within the MDCS cohort [10]. The index is based on the Swedish nutrition recommendations [11] for consumption of 6 dietary components: saturated fat $\leq 14$ energy $(\mathrm{E}) \%$, polyunsaturated fat $5-10$ $\mathrm{E} \%$, fish and shellfish $\geq 300 \mathrm{~g} /$ week, sucrose $\leq 10 \mathrm{E} \%$, dietary fiber $\geq 2.4 \mathrm{~g} / \mathrm{MJ}$ or $\geq 1 \mathrm{~g} / 100 \mathrm{kcal}$, and fruit and vegetables $\geq 400 \mathrm{~g} /$ day. $\mathrm{E} \%$ refers to daily energy percentage derived from each energy nutrient. Individuals who reached the recommendations were given 1 point for each component, with a maximum score of 6 points [10]. 
Lifestyle and Other Variables

Hypertension was defined as systolic blood pressure $\geq 140 \mathrm{~mm}$ $\mathrm{Hg}$ or diastolic blood pressure $\geq 90 \mathrm{~mm} \mathrm{Hg}$ or antihypertensive drug treatment. We have chosen to not include intermediary factors such as hypertension in the multivariable statistical models. Hypertension was nevertheless added in a sensitivity analysis in the final mutually adjusted multivariable statistical model. A smoking score was established, ranging from 0 (no smoking) to 4 (current smoking). Alcohol consumption was stratified into 6 groups. Education level was divided into 5 categories. Leisure-time physical activity level was expressed as metabolic equivalent of task hours per week based on intensity level and time spent on 17 different activities and was divided into 5 groups.

\section{Endpoint Ascertainment}

The MDCS cohort was followed from baseline (1991-1996) until 31 December 2016. Individuals with a first registered diagnosis of CAD were identified from the Swedish National Patient register and the Cause of Death Register by using the participants' civic registration numbers. Diagnoses are coded using a Swedish revision of the International Classification of Disease (ICD), version $8(432,00 ; 432,90)$, version 9 (443B), and version 10 (I65.2). $\mathrm{CAD}$ was defined by whenever the code for CAD first was registered, and symptomatic CAD when there was a $\geq 60 \%$ stenosis of the internal carotid artery on color Doppler ultrasound judged as responsible for an associated clinical event.

\section{Validation of CAD Diagnosis}

One hundred patients with a diagnosis of CAD (ICD 10; I65.2) were randomly selected for the validation procedure using patient record data. Among 100 patients, 57 had symptomatic ( $\geq 60 \%$ stenosis of the internal carotid artery on color Doppler ultrasound) CAD and 42 had asymptomatic CAD. The proportion of operated patients with symptomatic and asymptomatic ( $\geq 70 \%)$ CAD was $85.9 \%(49 / 57)$ and $14.3 \%(6 / 42)$, respectively. One patient had coronary artery disease and was misdiagnosed. The diagnosis of CAD was therefore confirmed in $99 \%$ of the validation sample and symptomatic CAD in $57 \%$.

\section{Statistical Analysis}

Baseline characteristics were expressed as median and interquartile range (IQR) or mean and standard deviation (SD) for continuous variables, and as total count and percentage for categorical variables. In order to address confounding in baseline characteristics, hazard ratios (HRs) with 95\% CI were calculated using the Cox regression analysis. The time scale was defined as years of follow-up. HRs for hypertension and lifestyle factors were adjusted for age and sex. HRs for diet variables were adjusted for age, sex, diet assessment method, season, and energy intake. HRs were calculated for adherence to each dietary recommendation. These variables were adjusted for age, sex, total energy intake, diet assessment method, and season in the basic model. In the multivariable model, the modifiable variables such as alcohol consumption, physical activity, smoking status, education level, and BMI were added. Each of the 6 dietary components included in the mutually adjusted model were adjusted for the variables in the multivariable model, as well as for the remaining 5 diet quality index components. Statistical analyses were performed using IBM SPSS Statistics, version 26 (SPSS, Chicago, IL, USA), and the chosen level of statistical significance was $p<0.05$.

Diet and Lifestyle as Risk Factors for Carotid Artery Disease

\section{Results}

\section{Demographic Baseline Characteristics}

During a median of 21.8 years (IQR 4.9) of follow-up, 469 out of $25,952(1.8 \%)$ patients were diagnosed with CAD. Baseline characteristics of those who developed CAD and those who did not are presented in Table 1. Individuals with hypertension had a higher risk for CAD (HR 2.20, 95\% CI: $1.78-2.72 ; p<0.001$ ). There was an increased risk for $\mathrm{CAD}$ among those who recently quit smoking (HR 1.52, 95\% CI: 1.12-2.07; $p=0.007$ ). A higher proportion of participants in the 2 quintiles with lower alcohol intake were seen in the group with no CAD.

\section{Adherence to Diet Quality Index Components and CAD Risk}

Individuals who adhered to the recommended intakes of the 6 different diet components did not show a significantly lower risk of CAD in the basic model or the multivariable model. 897 (3.5\%) and $770(3.0 \%)$ individuals had the highest (6) and the lowest (0) scores, respectively. There was no clear association between high diet score and incidence of CAD in the basic model ( $\mathrm{HR}=0.954$, 95\% CI: $0.893-1.020 ; p=0.169)$, the multivariable model $(\mathrm{HR}=0.969,95 \% \mathrm{CI}: 0.904-1.038 ; p=0.367)$, or the mutually adjusted multivariable model $(\mathrm{HR}=0.908,95 \% \mathrm{CI}$ : $0.811-1.017 ; p=0.095)$. There was a trend of decreased risk for reached recommendation of vegetables and fruit (HR 0.76, 95\% CI: 0.56-1.03; $p=0.080$ ) in the mutually adjusted multivariable model, but not for fiber intake (HR 0.77, 95\% CI: 0.56-1.06; $p=0.105$ ) (Table 2).

In a sensitivity analysis, hypertension was added into the mutually adjusted multivariable model, still resulting in a trend (HR 0.75, 95\% CI: $0.55-1.02$; $p=0.069$ ) for association between reached recommendation for vegetables and fruit and incident CAD. Hypertension was found to be an independent variable associated with incident CAD in the same model (HR 2.15, 95\% CI: 1.73-2.66; $p<$ 0.001 ).

\section{Discussion}

The main finding in this population-based prospective cohort study with a median follow-up of 21.8 years was a trend toward a protective effect of adherence to recommended levels of fruit and vegetable on risk of incident CAD. Similarly, fruit and vegetables have been found to reduce the incidence of peripheral artery disease [12], CV events [13], and abdominal aortic aneurysm [14] in the 
Table 1. Baseline characteristics of study participants with and without incident CAD in the MDCS cohort

\begin{tabular}{|c|c|c|c|c|}
\hline & $\begin{array}{l}\text { Incident CAD } \\
(n=469)\end{array}$ & $\begin{array}{l}\text { No CAD } \\
(n=25,483)\end{array}$ & $p$ value & $\begin{array}{l}\text { HR } \\
(95 \% \mathrm{CI})\end{array}$ \\
\hline Male sex, $n(\%)$ & $176(37.5)$ & $9,972(39.1)$ & 0.489 & $0.94(0.78-1.13)$ \\
\hline Age, years & $56.5(12.7)$ & $57.8(13.1)$ & 0.029 & $0.99(0.98-1.00)$ \\
\hline Total energy intake, kcal/day & $2,297.6(618.1)$ & $2,271.9(653.5)$ & 0.388 & $1.00(1.00-1.00)$ \\
\hline $\mathrm{BMI}, \mathrm{kg} / \mathrm{m}^{2}$ & $25.0(3.8)$ & $25.8(4.0)$ & $<0.001$ & $0.95(0.93-0.98)$ \\
\hline Hypertension, \% & $353(75.4)$ & $15,346(60.4)$ & $<0.001$ & $2.20(1.78-2.72)$ \\
\hline Zero-consumers & $42(9)$ & $1,644(6.5)$ & 0.176 & 1 (ref) \\
\hline$<0.9, \mathrm{~g} /$ day for women $/<3.4$, g/day for men & $73(15.6)$ & $4,766(18.7)$ & 0.007 & $0.59(0.41-0.87)$ \\
\hline $0.9-4.3, \mathrm{~g} /$ day for women $/ 3.4-9.1, \mathrm{~g} /$ day for men & $85(18.1)$ & $4,794(18.8)$ & 0.046 & $0.69(0.47-0.99)$ \\
\hline $4.4-8.1, \mathrm{~g} /$ day for women/9.2-15.7, g/day for men & $92(19.6)$ & $4,771(18.7)$ & 0.116 & $0.75(0.52-1.08)$ \\
\hline $8.2-14.0, \mathrm{~g} /$ day for women/15.7-25.7, g/day for men & $86(18.3)$ & $4,767(18.7)$ & 0.044 & $0.68(0.47-0.99)$ \\
\hline$>14.0, \mathrm{~g} /$ day for women/>25.7, g/day for men & $91(19.4)$ & $4,741(18.6)$ & 0.069 & $0.71(0.49-1.03)$ \\
\hline \multicolumn{5}{|l|}{ Smoking score, $n(\%)$} \\
\hline No smoking since $12-24$ years & $67(15.0)$ & $3,306(13.6)$ & 0.175 & $1.22(0.92-1.63)$ \\
\hline No smoking since $1-11$ years & $55(12.3)$ & $2,202(9.1)$ & 0.007 & $1.52(1.12-2.07)$ \\
\hline Currently smoking & $116(26.0)$ & $6,158(25.4)$ & 0.397 & $1.11(0.87-1.41)$ \\
\hline \multicolumn{5}{|l|}{ Leisure-time physical activity, $n(\%)$} \\
\hline$<7.5 \mathrm{MET}-\mathrm{h} /$ week & $34(7.3)$ & $2,476(9.8)$ & 0.340 & 1 (ref) \\
\hline 7.5-15.0 MET-h/week & $76(16.2)$ & $3,785(15.0)$ & 0.067 & $1.46(0.97-2.19)$ \\
\hline 15.1-25.0 MET-h/week & $106(22.6)$ & $5,817(23.0)$ & 0.163 & $1.32(0.89-1.94)$ \\
\hline 25.1-50.0 MET-h/week & $170(36.3)$ & $9,174(36.3)$ & 0.102 & $1.36(0.94-1.97)$ \\
\hline >50.0 MET-h/week & $82(17.5)$ & $4,051(16.0)$ & 0.046 & $1.50(1.01-2.24)$ \\
\hline \multicolumn{5}{|l|}{ Educational level, $n(\%)$} \\
\hline Less than 9 years & $181(38.6)$ & $10,663(42.0)$ & 0.582 & 1 (ref) \\
\hline Sucrose, E\% & $8.59(3.33)$ & $8.50(3.54)$ & 0.738 & $1.00(0.98-1.03)$ \\
\hline Fiber, g/MJ & $2.19(0.64)$ & $2.22(0.65)$ & 0.410 & $0.94(0.81-1.09)$ \\
\hline Vegetables and fruit, $\mathrm{g} / 1,000 \mathrm{kcal}$ & $338.02(206.37)$ & $347.21(230.08)$ & 0.646 & $1.00(1.00-1.00)$ \\
\hline Fish and shellfish, g/week & $277.00(290.18)$ & $277.09(290.01)$ & 0.790 & $1.00(1.00-1.00)$ \\
\hline
\end{tabular}

Data are $n(\%)$, mean (SD), or median (IQR). HR for hypertension and lifestyle factors adjusted for age and sex; HR for diet variables adjusted for age, sex, diet assessment method, season, and energy intake. E, energy; MET, metabolic equivalent of task; MJ, megajoules; $\mathrm{CAD}$, carotid artery disease; IQR, interquartile range; HR, hazard ratio.

same cohort. Only one other prospective longitudinal cohort study was found to specifically address the association between intake of vegetables and CAD, which showed an inverse association of vegetable nitrate intake with carotid atherosclerosis and ischemic cerebrovascular disease in older women after a follow-up of 14.5 years [15].
One randomized controlled trial aiming at reducing total fat intake and encouraging increased fruit, vegetables, and grains showed no change in the risk for incident CAD at 8.3 years of follow-up among postmenopausal women [16]. The study was originally designed to test the hypothesis that dietary intervention would reduce $\mathrm{CV}$ disease 
Table 2. HR and 95\% CI for incident CAD by adherence to diet quality index components

\begin{tabular}{|c|c|c|}
\hline Dietary components & Nonadherence & Adherence \\
\hline Saturated fat, $\%$ & $\geq 14 \mathrm{E}$ & $\leq 14 \mathrm{E}$ \\
\hline Incident CAD & $141 / 8,011$ & $364 / 19,139$ \\
\hline Basic model $^{\mathrm{a}}$ & $1.00(1.00-1.00)$ & $0.92(0.75-1.13)$ \\
\hline Multivariable model ${ }^{\mathrm{b}}$ & $1.00(1.00-1.00)$ & $0.96(0.77-1.18)$ \\
\hline Mutually adjusted multivariable $\operatorname{model}^{\mathrm{c}}$ & $1.00(1.00-1.00)$ & $0.97(0.72-1.29)$ \\
\hline Polyunsaturated fat, \% & $<5 \mathrm{E}$ or $>10 \mathrm{E}$ & $5-10 \mathrm{E}$ \\
\hline Incident CAD & $357 / 18,778$ & $148 / 8,372$ \\
\hline Basic model $^{\mathrm{a}}$ & $1.00(1.00-1.00)$ & $1.10(0.90-1.35)$ \\
\hline Multivariable model ${ }^{\mathrm{b}}$ & $1.00(1.00-1.00)$ & $1.09(0.89-1.34)$ \\
\hline Mutually adjusted multivariable model $^{\mathrm{c}}$ & $1.00(1.00-1.00)$ & $1.08(0.85-1.38)$ \\
\hline Sucrose, \% & $\geq 10 \mathrm{E}$ & $\leq 10 \mathrm{E}$ \\
\hline Incident CAD & $150 / 7,626$ & $355 / 19,524$ \\
\hline Basic model $^{\mathrm{a}}$ & $1.00(1.00-1.00)$ & $0.91(0.75-1.11)$ \\
\hline Multivariable model ${ }^{\mathrm{b}}$ & $1.00(1.00-1.00)$ & $0.93(0.76-1.14)$ \\
\hline Mutually adjusted multivariable model ${ }^{c}$ & $1.00(1.00-1.00)$ & $0.84(0.61-1.15)$ \\
\hline Fiber, g/MJ & $\leq 2.4$ & $\geq 2.4$ \\
\hline Incident CAD & $353 / 18,075$ & $152 / 9,075$ \\
\hline Basic model $^{\mathrm{a}}$ & $1.00(1.00-1.00)$ & $0.84(0.69-1.03)$ \\
\hline Multivariable model $^{\mathrm{b}}$ & $1.00(1.00-1.00)$ & $0.86(0.70-1.07)$ \\
\hline Mutually adjusted multivariable model $^{\mathrm{c}}$ & $1.00(1.00-1.00)$ & $0.77(0.56-1.06)$ \\
\hline Vegetables and fruit, g/day & $\leq 400$ & $\geq 400$ \\
\hline Incident CAD & $320 / 16,819$ & $185 / 10,331$ \\
\hline Basic model ${ }^{\mathrm{a}}$ & $1.00(1.00-1.00)$ & $0.88(0.72-1.06)$ \\
\hline Multivariable model ${ }^{\mathrm{b}}$ & $1.00(1.00-1.00)$ & $0.90(0.74-1.10)$ \\
\hline Mutually adjusted multivariable $\operatorname{model}^{\mathrm{c}}$ & $1.00(1.00-1.00)$ & $0.76(0.56-1.03)$ \\
\hline Fish, g/week & $\leq 300$ & $\geq 300$ \\
\hline Incident CAD & $279 / 14,722$ & $226 / 12,428$ \\
\hline Basic model ${ }^{\mathrm{a}}$ & $1.00(1.00-1.00)$ & $0.97(0.81-1.17)$ \\
\hline Multivariable model ${ }^{\mathrm{b}}$ & $1.00(1.00-1.00)$ & $1.00(0.82-1.21)$ \\
\hline Mutually adjusted multivariable model $^{\mathrm{c}}$ & $1.00(1.00-1.00)$ & $0.92(0.69-1.21)$ \\
\hline
\end{tabular}

$\mathrm{CAD}$, carotid artery disease; HR, hazard ratio. ${ }^{\text {a }}$ Basic model: adjusted for age, sex, and total energy intake. ${ }^{b}$ Multivariable model: adjusted for age, sex, total energy intake, alcohol consumption, physical activity, smoking, education, and BMI. ${ }^{c}$ Mutually adjusted multivariable model: adjusted for age, sex, total energy intake, alcohol consumption, physical activity, smoking, education, BMI, and mutual adjustment for the 6 diet quality index components.

defined as coronary heart disease and stroke, and were thus beforehand underpowered to investigate the association between the dietary intervention and risk for CAD. In another randomized clinical trial involving 175 subjects at high CV risk adhered to a Mediterranean diet rich in plant-derived foods and whole grain, a regression or delayed progression of internal carotid artery intima thickness, carotid plaques, and atherosclerosis in general was found at 2.4 years of follow-up [17]. Patients with $\mathrm{CAD}$ and other atherosclerotic diseases have a high longterm all-cause and cardiac-related mortality [18] and ev- idence points to a diet rich in plant foods playing a major role in preventing atherosclerotic development regardless of localization in the vascular tree.

Several mechanisms have been suggested to explain the effect of plant foods on atherosclerotic development. Vitamin C has been shown to restore endothelial function through improved perfusion in atherosclerotic sites of vessels, hindering the atherosclerotic process. Another study on the MDCS cohort found vegetables and fruit to be protective against AAA. Fruit and vegetables are rich in antioxidants, which are believed to inhibit oxidative 
stress in the aortic wall, thereby reducing AAA progression. Furthermore, inorganic nitrate found in green leafy vegetables has been shown to act in a protective way, reducing high blood pressure and plaque development [14]. The fact that individuals with a higher intake of vegetables and fruit also may lead a healthier lifestyle in general, decreasing the risk of $\mathrm{CV}$ disease, should also be taken into consideration.

There is a risk of self-reported dietary and lifestyle habits being misreported to some extent, and these habits might have changed at follow-up. It is, however, unlikely that the middle-aged study participants markedly changed their dietary habits in their later stage of life. In a longitudinal study following middle-aged individuals attempting fat reduction showed that almost $70 \%$ had not altered their diet after 24 months [19]. In addition, individuals who might have altered their diet due to diabetes and CV disease were excluded at baseline. Moreover, the dietary assessment methods used have been shown to have high validity [10]. Furthermore, it could have been of value to perform ultrasound examinations at baseline and at follow-up in order to identify cases of asymptomatic CAD. Thereby patients with prevalent asymptomatic CAD could have been excluded at baseline, and also given a larger sample size of individuals with incident CAD at follow-up. Accuracy of data would have improved, and a larger endpoint sample size would have increased statistical power and reducing the risk of type 2 statistical error, perhaps turning trends to significant results.

The main strengths of the present study are the large sample size and the follow-up time of over 20 years. These are strong methodological factors making it possible to follow the middle-aged participants into older age, where the incidence of CAD peaks [20]. Registries were used to attain disease endpoints, which poses another significant strength as it ensured almost complete follow-up of the study participants. Furthermore, the validity of the CAD diagnosis was high, with confirmation of the diagnosis in $99 \%$ of the validation sample.
A systematic review of 20 studies found a general trend between diets rich in plant foods and decreased carotid intima thickness [21]. The study emphasized the need for long-term prospective studies on large populations to further investigate the effects of dietary patterns on CAD, which highlights the value of the present study.

In conclusion, the present study found a trend toward a protective effect of higher intake of vegetables and fruit against incident CAD. More prospective studies investigating the association between diet and $\mathrm{CAD}$ and stroke are needed to be able to give firm recommendations.

\section{Statement of Ethics}

The study was conducted ethically in accordance with the World Medical Association Declaration of Helsinki. Written and oral informed consent was obtained from the study participants and ethical clearance was obtained from the Regional Ethical Review Board in Lund, Sweden (Dnr \$ LU 51-90, 2007/166).

\section{Conflict of Interest Statement}

The authors have no conflicts of interest to declare.

\section{Funding Sources}

This research received a grant from the Swedish STROKE foundation.

\section{Author Contributions}

S.A. and A.J. conceived of the concept and design of the work. S.A. and A.J. performed the analysis and interpretation of data. A.J. drafted the work. S.A. revised it critically and approved the final version to be published. A.J. and S.A. agreed to be accountable for all aspects of the work.

\section{References}

1 World Population Prospects [Web Page]. United Nations DESA Population Division; [updated 28 August 2019; cited 22 April 2020. Available from: http://population.un.org/ $\mathrm{wpp} /]$.

2 Wilkins E, Wilson L, Wickramasinghe K, Bhatnagar P, Leal J, Luengo-Fernandez R, et al. European cardiovascular disease statistics. Report. Brussels: European Heart Network; 2017.
3 Henssge U, Hoffman A, Kavanagh S, Roughton M, Rudd A, Cloud G. National sentinel stroke clinical audit 2010: generic report. London: Royal College of Physicians; 2011.

4 Naylor AR. Why is the management of asymptomatic carotid disease so controversial? Surgeon. 2015;13(1):34-43.

5 Sirimarco G, Amarenco P, Labreuche J, Touboul PJ, Alberts M, Goto S, et al. Carotid atherosclerosis and risk of subsequent coronary event in outpatients with atherothrombosis. Stroke. 2013;44(2):373-9.

6 Naylor AR, Ricco JB, De Borst GJ, Debus S, De Haro J, Halliday A, et al. Editor's choicemanagement of atherosclerotic carotid and vertebral artery disease: 2017 clinical practice guidelines of the European society for vascular surgery (ESVS). Eur J Vasc Endovasc Surg. 2018;55(1):3-81. 
7 Mendis S. Global status report on noncummunicable diseases. Report. Geneva: WHO; 2014.

8 Malmö kost cancer och Malmö förebyggande medicin [web page]. Lund: Lund University; [updated 11 Nov 2019; cited 18 May 2020. Available from: https://www.malmo-kohorter.lu.se/malmo-kost-cancer-mkc].

9 Wirfält E, Mattisson I, Johansson U, Gullberg B, Wallström P, Berglund G. A methodological report from the Malmö diet and cancer study: development and evaluation of altered routines in dietary data processing. Nutr J. 2002;1:3.

10 Drake I, Gullberg B, Ericson U, Sonestedt E, Nilsson J, Wallström P, et al. Development of a diet quality index assessing adherence to the Swedish nutrition recommendations and dietary guidelines in the Malmö diet and cancer cohort. Public Health Nutr. 2011;14(5):83545.

11 Tetens I, Pedersen AN, Schwab U, Fogelholm M, Thorsdottir I, Gunnarsdottir I, et al. Nordic nutrition recommendations. Report. Copenhagen: Nordic Council of Ministers; 2014.

12 Kulezic A, Bergwall S, Fatemi S, Sonestedt E, Zarrouk M, Gottsäter A, et al. Healthy diet and fiber intake are associated with decreased risk of incident symptomatic peripheral artery disease: a prospective cohort study. Vasc Med. 2019;24(6):511-8.
13 Hlebowicz J, Drake I, Gullberg B, Sonestedt E, Wallström P, Persson M, et al. A high diet quality is associated with lower incidence of cardiovascular events in the Malmö diet and cancer cohort. PLoS One. 2013;8(8):e71095-

14 Bergwall S, Acosta S, Sonestedt E. Intake of fibre and plant foods and the risk of abdominal aortic aneurysm in a large prospective cohort study in Sweden. Eur J Nutr. 2020 Aug; 59(5):2047-56.

15 Bondonno CP, Blekkenhorst LC, Prince RL, Ivey KL, Lewis JR, Devine A, et al. Association of vegetable nitrate intake with carotid atherosclerosis and ischemic cerebrovascular disease in older women. Stroke. 2017;48(7): 1724-9.

16 Allison MA, Aragaki A, Eaton C, Li W, Van Horn L, Daviglus ML, et al. Effect of dietary modification on incident carotid artery disease in postmenopausal women: results from the women's health initiative dietary modification trial. Stroke. 2014;45(6):1748-56.
17 Sala-Vila A, Romero-Mamani ES, Gilabert R, Núñez I, de la Torre R, Corella D, et al. Changes in ultrasound-assessed carotid intima-media thickness and plaque with a Mediterranean diet: a substudy of the PREDIMED trial. Arterioscler Thromb Vasc Biol. 2014;34(2): 439-45.

18 Giannopoulos A, Kakkos S, Abbott A, Naylor AR, Richards T, Mikhailidis DP, et al. Longterm mortality in patients with asymptomatic carotid stenosis: implications for statin therapy. Eur J Vasc Endovasc Surg. 2015;50(5): 573-82.

19 Yusufov M, Paiva AL, Redding CA, Lipschitz JM, Gokbayrak NS, Greene G, et al. Fat reduction efforts: a 24-month longitudinal comparison of a large sample of maintainers, relapsers, and non-changers. Health Promot Pract. 2016;17(1):116-26.

20 Kelly-Hayes M. Influence of age and health behaviors on stroke risk: lessons from longitudinal studies. J Am Geriatr Soc. 2010; 58(Suppl 2):S325-8.

21 Bhat S, Mocciaro G, Ray S. The association of dietary patterns and carotid intima-media thickness: a synthesis of current evidence. Nutr Metab Cardiovasc Dis. 2019;29(12): 1273-87. 